\title{
Diffusion of Vitamin B12 Across a Mesoporous Metal Organic Framework
}

Veronica Valencia

University of South Florida, veronica8@mail.usf.edu

\author{
Advisors: \\ Thomas Bieske, Mathematics and Statistics \\ Shengqian Ma, Chemistry \\ Yao Chen, Chemistry \\ Scott Campbell, Chemical \& Biomedical Engineering \\ Problem Suggested By: Shengqian Ma
}

Follow this and additional works at: https://digitalcommons.usf.edu/ujmm

Part of the Materials Chemistry Commons, Mathematics Commons, and the Organic Chemistry Commons

UJMM is an open access journal, free to authors and readers, and relies on your support:

Donate Now

\section{Recommended Citation}

Valencia, Veronica (2013) "Diffusion of Vitamin B12 Across a Mesoporous Metal Organic Framework," Undergraduate Journal of Mathematical Modeling: One + Two: Vol. 5: Iss. 1, Article 3.

DOI: http://dx.doi.org/10.5038/2326-3652.5.1.3

Available at: https://digitalcommons.usf.edu/ujmm/vol5/iss $1 / 3$ 


\title{
Diffusion of Vitamin B12 Across a Mesoporous Metal Organic Framework
}

\begin{abstract}
We measure the rate of uptake and the rate of release of a Vitamin B12 solution (dissolved in water) at 2 different temperatures (room temperature and $37^{\circ} \mathrm{C}$ ) by the mesoporous metal organic framework TbMOF-100 at 1-hour intervals using a spectrophotometer. Using the Beer-Lambert law, we calculate the concentration of the stock solution based on the absorbance values obtained with the spectrophotometer. These values allow for the quantification of the initial rate of uptake and the rate of uptake at a random incubation time of the Vitamin B12 by the TbMOF-100. We also calculate the value of the coefficient of diffusion for this 2-phase system using Fick's laws of diffusion.
\end{abstract}

\section{Keywords}

Diffusion, Fick's Laws of Diffusion, Beer-Lambert Law 


\section{PROBLEM STATEMENT}

Study the kinetics of diffusion of biomolecules into new types of porous materials at different temperatures. Consider the initial rate of uptake, the diffusion rate, and the coefficient of diffusion for a 2-phase system consisting of TbMOF-100 and Vitamin B12 solution.

\section{Motivation}

Porous metal-organic frameworks (MOFs) are highly crystalline inorganic-organic hybrids, and they are constructed by assembling metal ions or metal-containing clusters known as secondary building units (SBUs) with multidentate organic ligands via coordination bonds into a three-dimensional structure. The research experiment conducted dealt with the applications of MOFs for biocatalysis, which according to the New Zealand Institute of Chemistry, can be broadly defined as the use of biological molecules usually enzymes to catalyze specific chemical reactions (Chowdhury, Hill and Whittaker). Due to their high porosity and nanospace inside the framework, MOFs show great promise in the field of biocatalysis as these mesoporous complexes can encapsulate proteins, enzymes, and vitamins within the framework. A study of the diffusion of vitamins into metal-organic frameworks is relevant, because it provides information that is necessary to determine the efficiency of encapsulating the vitamin within the mesoporous structure. A calculation of the diffusion coefficient of this two-phase system provides conclusive data on the rate of diffusion of the vitamin into the MOF structure. 


\section{MATHEMATICAL DESCRIPTION AND SOLUTION APPROACH}

To calculate the initial rate of uptake and the diffusion rate, the absorbance values calculated experimentally, were converted to actual concentrations according to the BeerLambert Law:

$$
A=\varepsilon l c
$$

Where $A$ is the absorbance, $l$ is the path length (equal to $1 \mathrm{~cm}$ ), $\varepsilon$ is the molar absorptivity constant $\left(2.65 \times 10^{4} \frac{\mathrm{L}}{\mathrm{Mol} \cdot \mathrm{cm}}\right)$, and $c$ is the concentration in Moles. Table 1 below provides both the absorbance values collected during the experiment, as well as the concentration of the solution calculated using the Beer-Lambert Law.

\begin{tabular}{|c|c|c|}
\hline $\begin{array}{c}\text { Incubation } \\
\text { Time (in } \\
\text { hours) }\end{array}$ & $\begin{array}{c}\text { Vitamin B12 } \\
\text { Solution } \\
\text { Absorbance }\end{array}$ & $\begin{array}{c}\text { Vitamin B12 } \\
\text { Solution } \\
\text { concentration } \\
\text { (in } \mathbf{~ m M )}\end{array}$ \\
\hline $\mathbf{0}$ & 0.5919 & 2.23 \\
\hline $\mathbf{1}$ & 0.5057 & 1.91 \\
\hline $\mathbf{2}$ & 0.5061 & 1.91 \\
\hline $\mathbf{3}$ & 0.4715 & 1.78 \\
\hline $\mathbf{4}$ & 0.4684 & 1.77 \\
\hline $\mathbf{5}$ & 0.4406 & 1.66 \\
\hline $\mathbf{6}$ & 0.4350 & 1.64 \\
\hline $\mathbf{1 9 . 3}$ & 0.3451 & 1.3 \\
\hline
\end{tabular}

\begin{tabular}{|c|c|}
\hline $\begin{array}{c}\text { Incubation } \\
\text { Time } \\
\text { (in hours) }\end{array}$ & $\begin{array}{c}\text { Concentration } \\
\text { absorbed by } \\
\text { TbMOF-100 } \\
\text { (in mM) }\end{array}$ \\
\hline $\mathbf{0}$ & 0 \\
\hline $\mathbf{1}$ & 0.32 \\
\hline $\mathbf{2}$ & 0.32 \\
\hline $\mathbf{3}$ & 0.45 \\
\hline $\mathbf{4}$ & 0.46 \\
\hline $\mathbf{5}$ & 0.57 \\
\hline $\mathbf{6}$ & 0.59 \\
\hline $\mathbf{1 9 . 3}$ & 0.93 \\
\hline
\end{tabular}

Table 1: Vitamin B12 Solution Absorbance and Concentration

Table 2: Concentration Absorbed by TbMOF-100

Moreover, it was necessary to calculate the concentration absorbed by the TbMOF-100 at each time interval. The results are summarized in Table 2. 
We plot the data in Table 2 and determine a polynomial approximation for the line of best fit.

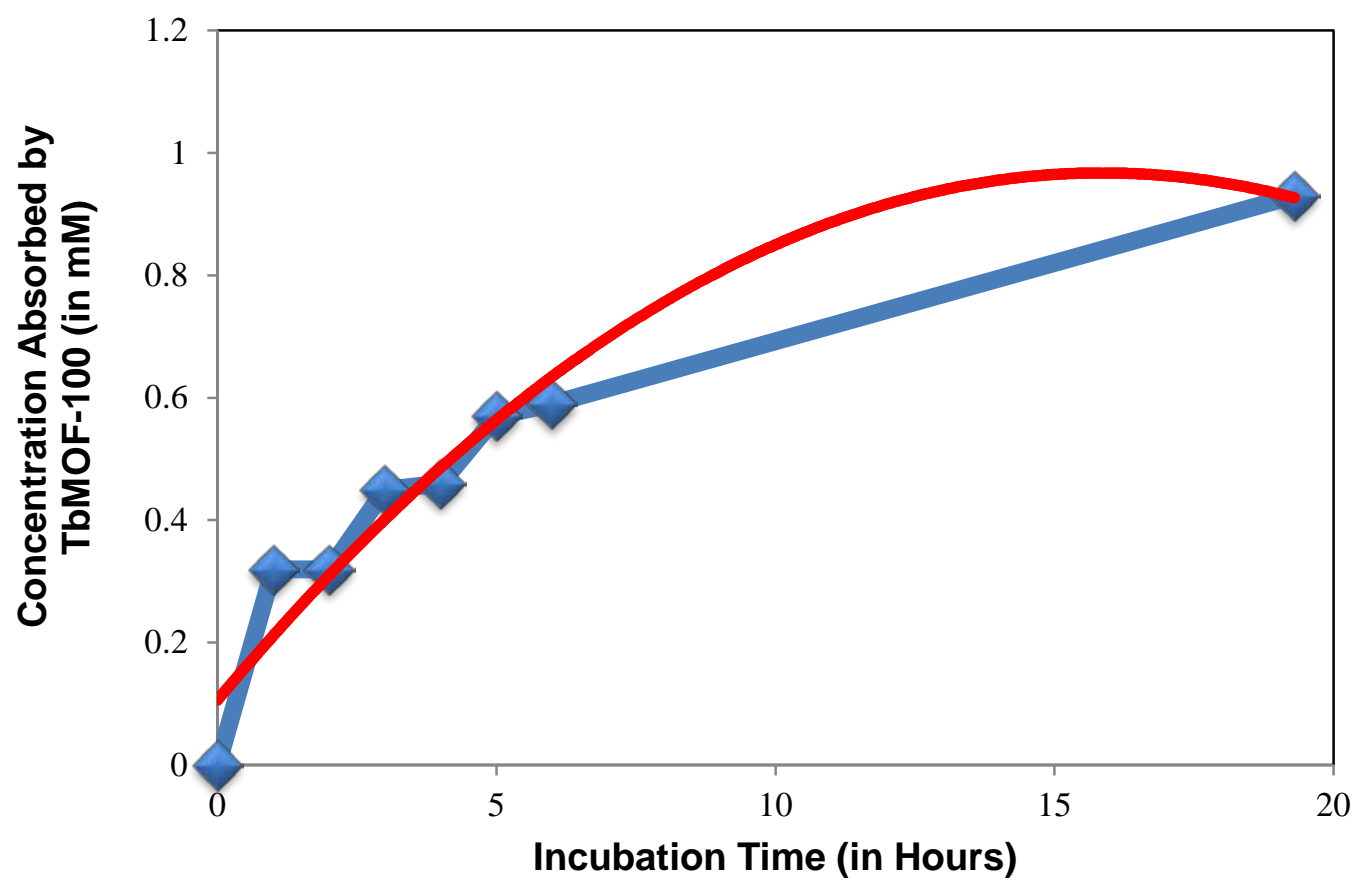

Plot 1: Measured (blue) TbMOF-100 uptake profile of Vitamin B12 and best-fit parabola (red)

The equation for the best-fit parabola pictured in Plot 1 is given by:

$$
F(x)=-0.0034 x^{2}+0.1086 x+0.1063
$$

where $x$ is incubation time in hours. Using (2) it is now possible to determine both the initial rate of reaction and the instantaneous reaction rate at any given time. The shape of the above graph suggests that initial reaction rate is fairly constant over the first few hours; therefore the initial rate of the reaction can be calculated by differentiating (2) and evaluating $F^{\prime}(x)$ at one of the initial data points. Differentiating (2) yields:

$$
F^{\prime}(x)=-0.0068 x+0.1086
$$

For our purposes we used the data point $(3,0.45)$, thus the initial rate of uptake is given by:

$$
F^{\prime}(3)=0.0881 \mathrm{mM} / \text { hour }
$$


The reaction rate at a given point in the uptake profile can be calculated in a similar manner. For example, the reaction rate at 7 hours of incubation time is determined by:

$$
F^{\prime}(7)=0.0607 \mathrm{mM} / \text { hour }
$$

According to the book Transport Phenomena in Biological Systems, "the diffusion coefficient of a molecule diffusing through a mesoporous structure can be found from measurements of the concentration of the solution as a function of time" (Chowdhury, Hill and Whittaker). The measurements are fit to a model for diffusion from a well-mixed bath into a sphere of radius $R$. In this model, we assume that the geometrical structure of the TbMOF-100 is a sphere, which in reality is not, as can be seen from Figure 1 below.
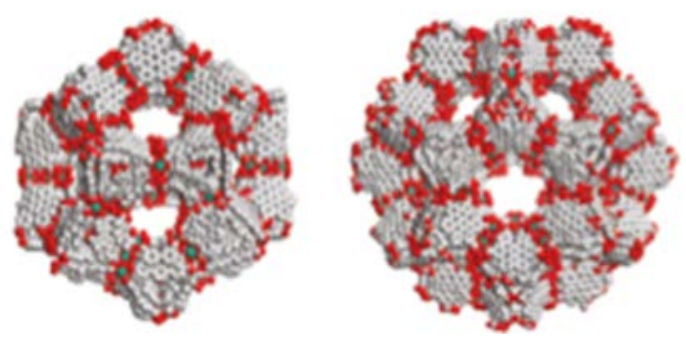

Figure 1: Molecular structure of TbMOF-100

An approximation of the true value of the diffusion coefficient for this 2-phase system can be calculated through the use of the following formula:

$$
\theta_{t}=\frac{\beta}{\beta+1}+\sum_{i=1}^{\infty} \frac{6 \beta e^{-\lambda_{i}^{2} \tau}}{9(1+\beta)+\beta^{2} \lambda_{i}^{2}}
$$

In this model, the equation has been cast in dimensionless form through the use of Laplace transforms. The variables in this equation are defined as follows:

$$
\theta_{f}=\frac{C_{t}}{C_{0}}, \beta=\frac{V_{f}}{\Phi V_{b}}, \text { and } \tau=\frac{t D_{e f f}}{R^{2}}
$$

where $C_{t}$ is the concentration in the solution in water at time $t, C_{0}$ is the initial concentration of the Vitamin B12 solution, $V_{f}$ is the volume of the solution, $V_{b}$ is the volume of the TbMOF-100 
crystals, $D_{e f f}$ is the diffusion coefficient of the solution, and $R$ is the radius of the crystal structure. In this case we assume $\Phi$, the partition coefficient, is equal to 1 . Furthermore, in this model, $\lambda_{i}$ are the solutions to the equation:

$$
\tan \lambda_{i}=\frac{3 \lambda_{i}}{3+\beta \lambda_{i}^{2}}
$$

Once $\beta$ is determined, $\lambda_{i}$ can be found as the zeros of the function

$$
G(s)=\tan (s)-\frac{3 s}{3+\beta s^{2}} .
$$

From (6) and (7) we get

$$
\frac{C_{t}}{C_{o}}=\frac{V_{f} / V_{b}}{V_{f} / V_{b}+1}+\sum_{i=1}^{\infty} \frac{6\left(\frac{V_{f}}{V_{b}}\right) e^{\left(-\lambda_{i}^{t} \frac{t D_{e f f}}{R^{2}}\right)}}{9\left(1+\frac{V_{f}}{V_{b}}\right)+\left(\frac{V_{f}}{V_{b}}\right)^{2} \lambda_{i}^{2}} .
$$

and as $t \rightarrow \infty, e^{-\lambda_{i}^{2} \frac{t D_{e f f}}{R^{2}}} \rightarrow 0$, thus when the system reaches equilibrium the infinite sum on the right hand side tends towards zero, so $\theta_{t} \approx \frac{\beta}{\beta+1}$. We assume that the concentration after 19.3 hours of incubation is the same as the concentration at equilibrium. This gives:

$$
\frac{1.3 m M}{2.23 m M}=\frac{\beta}{\beta+1} .
$$

And we conclude that

$$
\beta=1.39785
$$

which allows us to use $G(s)$ from (9) to find the values of $\lambda_{i}$. The plot of $G(s)$ shows the different values for $\lambda_{i}$ and the values are given in Table 3 . 


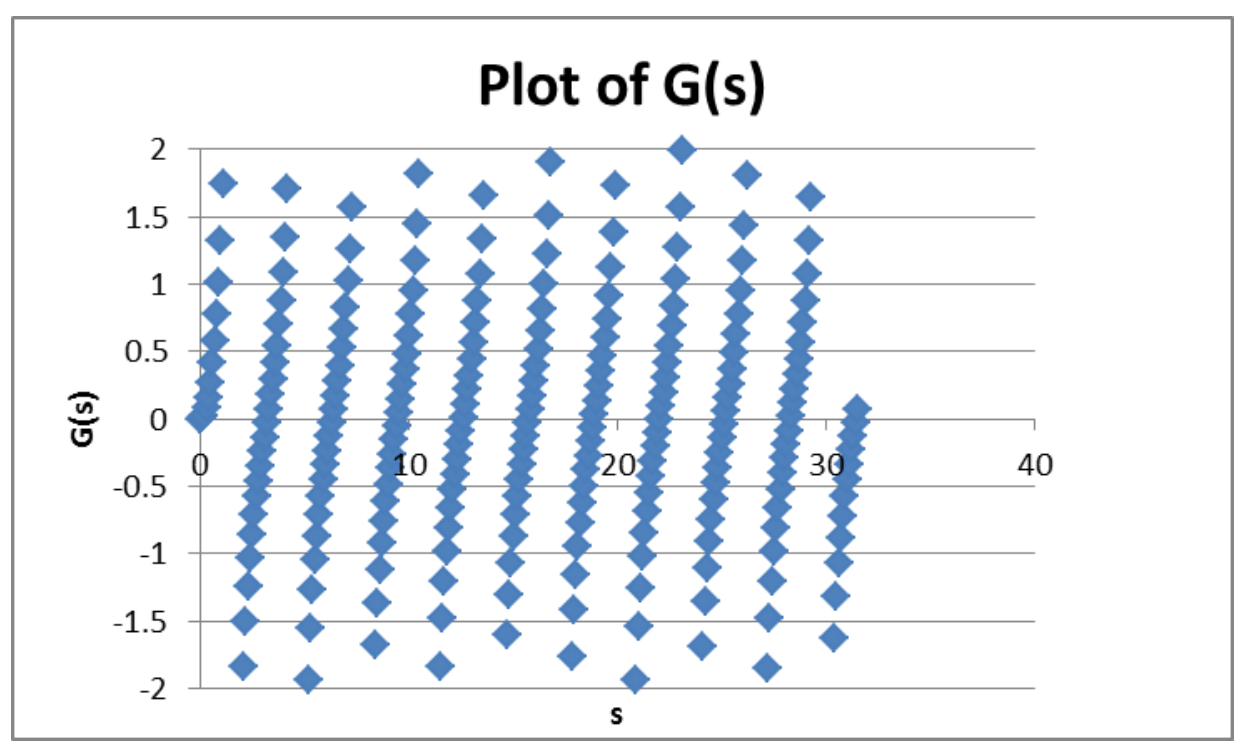

\begin{tabular}{|cc|}
\hline $\boldsymbol{i}$ & $\lambda_{i}$ \\
\hline $\mathbf{1}$ & 3.232 \\
\hline $\mathbf{2}$ & 6.331 \\
\hline $\mathbf{3}$ & 9.457 \\
\hline $\mathbf{4}$ & 12.591 \\
\hline $\mathbf{5}$ & 15.728 \\
\hline $\mathbf{6}$ & 18.865 \\
\hline $\mathbf{7}$ & 22.004 \\
\hline $\mathbf{8}$ & 25.217 \\
\hline
\end{tabular}

Plot 2: Numerical plot of $G(s)$ as defined by Equation (9) and table of the first few approximations of $G(s)=0$.

Now with the value of $\beta$ we are in position to use (10) to calculate $D_{e f f}$. Substituting for the known constants in (10) and using the data collected after 3 hours of incubation we have:

$$
\begin{gathered}
\frac{1.78 m M}{2.23 m M}=\frac{1.39785}{1.39785+1}+\sum_{i=1}^{\infty} \frac{6(1.39785) e^{\left(-\lambda_{i}^{2} \frac{(3) D_{e f f}}{(100 ~ n m)^{2}}\right)}}{9(1+1.39785)+(1.39785)^{2} \lambda_{i}^{2}} \\
0.79821=0.58296+\sum_{i=1}^{\infty} \frac{(8.3871) e^{\left(3 * 10^{-4}\right)\left(-\lambda_{i}^{2}\right) D_{e f f}}}{(21.58065)+(1.95398) \lambda_{i}^{2}} \\
0.21525=\sum_{i=1}^{\infty} \frac{(8.3871)(1.0003)^{\left(-\lambda_{i}^{2}\right) D_{e f f}}}{(21.58065)+(1.95398) \lambda_{i}^{2}}
\end{gathered}
$$

To calculate the true value of the coefficient of diffusion we used the known $\lambda_{i}$ s to approximate the value of the infinite sum. Thus (13) gives:

$$
D_{e f f}=66 \mathrm{~nm}^{2} / \mathrm{hr}
$$

The results of this method are summarized in the appendix. 


\section{DISCUSSION}

The objective of this project was to calculate the initial rate of uptake, the rate of uptake at a random incubation time, and the coefficient of diffusion for this 2-phase system. The objectives of this project were met. The initial rate of uptake was calculated to be $0.088 \mathrm{mM} / \mathrm{hr}$ using the first derivative of the best-fit line for the data. The rate of the uptake at the random incubation time (at 7 hours of incubation) was calculated to be $0.061 \mathrm{mM} / \mathrm{hr}$. These calculations were expected, since rates of diffusion are always highest at the beginning of the uptake profile and decrease, until the system reaches equilibrium. This characteristic is also supported by the graphical representation of the data. In addition, the coefficient of diffusion for this 2-phase system was found to be $66 \mathrm{~nm}^{2} / \mathrm{hr}$, which was also expected, since this positive value confirms that diffusion of the Vitamin B12 into the mesoporous material did indeed occur.

\section{CONCLUSION AND RECOMMENDATIONS}

In this experiment, the initial rate of uptake, reaction rate, and coefficient of diffusion were calculated for the diffusion of a Vitamin B12 solution into a mesoporous crystal called TbMOF-100. As expected, the initial rate of uptake was higher than the rate of uptake at later incubation times. In addition, the coefficient of diffusion was calculated to be $66 \mathrm{~nm}^{2} /$ hour which confirms the diffusion of the Vitamin B12 into the TbMOF-100. This indicates that the TbMOF-100 is a porous material as its value for the coefficient of diffusion can be explained by its porous structure, which allows for the capture of the Vitamin B12 inside its cages.

Considering that the model used to calculate the coefficient of diffusion is only an approximation, several recommendations could be given to provide a more accurate result for the 
value of the coefficient of diffusion for this system. Since the model is designed to measure the coefficient of diffusion of a well-mixed bath into a spherical structure, it would be useful to conduct the experiment to measure the uptake profile on a sonicator to keep the Vitamin B12 solution constantly mixed. Another recommendation would be to construct a new model to compute the coefficient of diffusion, which takes into account the non-spherical structure of TbMOF-100. 


\section{NOMENCLATURE}

\begin{tabular}{|clc|}
\hline Symbol & Definition & Units \\
\hline $\boldsymbol{E}$ & Absorbance & N/A \\
\hline $\boldsymbol{l}$ & Molar absorptivity constant & $\mathrm{Mol} \cdot \mathrm{cm}$ \\
\hline $\boldsymbol{c}$ & Path Length & $\mathrm{cm}$ \\
\hline $\boldsymbol{C}_{\boldsymbol{t}}$ & Concentration & $\mathrm{mM}$ \\
\hline $\boldsymbol{C}_{\mathbf{0}}$ & Initial concentration of the solution & $\mathrm{mM}$ \\
\hline $\boldsymbol{V}_{\boldsymbol{f}}$ & Volume of the solution & $\mathrm{mM}$ \\
\hline $\boldsymbol{V}_{\boldsymbol{b}}$ & Volume of the TbMOF-100 crystals & $\mathrm{mL}$ \\
\hline $\boldsymbol{D}_{\boldsymbol{e} \boldsymbol{f}}$ & Diffusion coefficient of the solution & $\mathrm{nm} / \mathrm{hour}$ \\
\hline $\boldsymbol{R}$ & Radius of the crystal structure & $\mathrm{nm}$ \\
\hline $\boldsymbol{t}$ & Incubation time & $\mathrm{hours}$ \\
\hline
\end{tabular}

\section{REFERENCES}

Chowdhury, Mohammad, David Hill and Andrew Whittaker. "Vitamin B12 Release from P(Hema-co-Thema) in Water and SBF: A Model Drug Release Study." Aust J Chem (2005): 451-456.

Larson, Ron, Robert Hostetler and Bruce Edwards. Calculus. 8th Edition. Boston, MA: Houghton Mifflin Company, 2005.

Saul, David J. Biocatalysis: Industrial Enzymesand the Exploitation of Micro-Organisms. New Zealand Institute of Chemistry, 2010.

Truskey, George A, Fan Yua and David Katz. Transport Phenomena in Biological Systems. Pearson Prentice Hall Engineering, 2004. 


\section{APPENDIX}

From (13) we have:

$$
0.21525=\sum_{i=1}^{\infty} \frac{(8.3871)(1.0003)^{\left(-\lambda_{i}^{2}\right) D_{e f f}}}{(21.58065)+(1.95398) \lambda_{i}^{2}}
$$

Thus:

$$
\begin{aligned}
0.21525=\frac{8.3871(0.99687)^{D_{e f f}}}{41.99158}+\frac{8.3871(0.98805)^{D_{e f f}}}{99.89922}+\frac{8.3871(0.97353)^{D_{e f f}}}{112.96848} \\
+\frac{8.3871(0.95356)^{D_{e f f}}}{331.35151}+\frac{8.3871(0.92849)^{D_{e f f}}}{504.93665}+\frac{8.3871(0.89875)^{D_{e f f}}}{716.97912} \\
+\frac{8.3871(0.86482)^{D_{e f f}}}{967.65090}
\end{aligned}
$$

Dividing 8.3871 from both sides gives:

$$
\begin{aligned}
0.025664= & \frac{(0.99687)^{D_{e f f}}}{41.99158}+\frac{(0.98805)^{D_{\text {eff }}}}{99.89922}+\frac{(0.97353)^{D_{\text {eff }}}}{112.96848}+\frac{(0.95356)^{D_{\text {eff }}}}{331.35151} \\
& +\frac{(0.92849)^{D_{\text {eff }}}}{504.93665}+\frac{(0.89875)^{D_{\text {eff }}}}{716.97912}+\frac{(0.86482)^{D_{\text {eff }}}}{967.65090}
\end{aligned}
$$

The best value for $D_{\text {eff }}$ was found to be $66 \mathrm{~nm}^{2} / \mathrm{hr}$. We checked this value with the data obtained from the fifth hour of incubation to test the quality of our solution. Thus:

$$
\begin{gathered}
\frac{1.66 m M}{2.23 m M}=\frac{1.39785}{1.39785+1}+\sum_{i=1}^{\infty} \frac{6(1.39785) e^{\left(-\lambda_{i}^{2} \frac{(5) D_{e f f}}{(100 n m)^{2}}\right)}}{9(1+1.39785)+(1.39785)^{2} \lambda_{i}^{2}} \\
0.74439=0.58296+\sum_{i=1}^{\infty} \frac{(8.3871) e^{\left(5 * 10^{-4}\right)\left(-\lambda_{i}^{2}\right) D_{e f f}}}{(21.58065)+(1.95398) \lambda_{i}^{2}} \\
0.16143=\sum_{i=1}^{\infty} \frac{(8.3871)(1.0005)^{\left(-\lambda_{i}^{2}\right) D_{e f f}}}{(21.58065)+(1.95398) \lambda_{i}^{2}}
\end{gathered}
$$


Plugging in seven of the first $\lambda_{i}$ (the contribution to the sum is negligible for $i>7$ ) the sum on the right hand side becomes:

$\frac{5.94214}{41.99158}+\frac{2.23521}{99.89922}+\frac{0.43871}{112.96848}+\frac{0.044887}{331.35151}+\frac{0.0023948}{504.93665}+\frac{0.000066742}{716.97912}+\frac{0.00000096888}{967.65090} \approx 0.167906$

Hence the error is small so we conclude our value is accurate. 